\title{
An Improved Method of Linear Travel-Time Interpolation Ray Tracing Algorithm
}

\author{
W. HAO-QUAN* \\ Key Laboratory of Instrument Science \& Dynamic Measurement (North University of China) \\ Ministry of Education, National Key Laboratory of Science and Technology on Electronic Test \\ and Measurement, Taiyuan, Shanxi 030051, China
}

(Received September 23, 2009; revised version February 1, 2010; in final form May 5, 2010)

\begin{abstract}
The linear travel-time interpolation algorithm is improved by the method of ray tracing based on finite difference scheme of eikonal equation. It can simulate the more complex medium. Using the algorithm to calculate travel-time and paths of tracing rays, it is more rapid and accurate than the other conventional methods. The linear travel-time interpolation ray tracing algorithm has a drawback: rays cannot go round the low velocity area if the launching point, the receiving point and the low velocity area distribute on the same column (row). Aiming at the drawback, an improved algorithm is proposed on the basis of researching reconstruction theories. The given algorithm adopts a cross-scanning strategy to carry out forward processing and uses discrete points as the secondary sources to simplify backward processing. The numerical simulation experiments show that the rays can go round the low velocity areas located in the same column (row) of the launching points and the receiving points. Rays scan over the global region only once.
\end{abstract}

PACS numbers: 07.05.Pj, 42.30.Wb. 43.60. + d

\section{Introduction}

In ultrasound computed tomography, sonic velocity of points on a tomography surface is not equal. Ultrasound traveling paths are curves reflecting the non-uniformity of materials. Based on these similarities with earthquake wave people began to apply ray tracing methods in earthquake tomography to ultrasound tomography [1-3].

In recent years, ray tracing global methods which consider travel-time and paths of all discrete points have aroused widespread interest. The rationale of methods still is eikonal equation, Fermat principle and Huygens principle. They can calculate the travel-time accurately in complex models [4-7].

The conventional ray tracing methods generally include shooting methods of initial value problems and bending methods of boundary value problems. However, many problems exist in these methods. For example, it is difficult to deal with great velocity variation in medium and to work out the global minimum travel-time in multi-valued travel-time. It also has low computational efficiency. Even the density of rays covered in shadow areas is insufficient [8-10].

Asakawa (1993) proposed a new ray tracing algorithm based on the linear travel-time interpolation (LTI). The

* e-mail: wang_haoquan@nuc.edu.cn experiments indicate that this global algorithm can simulate the more complex medium. Using the algorithm to calculate travel-time and paths of tracing rays, it is more rapid and accurate than the other conventional methods [11]. The linear travel-time interpolation algorithm is improved by the method of ray tracing based on finite difference scheme of eikonal equation. At the same time, Asakawa also theoretically proved that the LTI algorithm is an advanced form of the method which is proposed by Vidale [12].

\section{Travel-time and path tracing algorithm}

Supposing the entire rectangular region is a grid, a ray goes through the grid boundary and arrives at the point $C$ between $A_{1}$ and $A_{2}$, as shown in Fig. 1. The formula calculating the local minimum travel-time will be deduced in the following [11].

Assuming that the grid's slowness is $S$, the length between the discrete points $A_{1}$ and $A_{2}$ is $L$, the travel-time of $A_{1}(x, y)$ and $A_{2}(x, y)$ is $T_{A 1}$ and $T_{A 2}$, respectively, $C(r, y)$ is the intersection of $A_{1} A_{2}$ and a ray which is from $P$, the travel-time from $A_{1}$ to $C$ can be calculated

$$
T_{C}=T_{A 1}+\frac{\left(x_{C}-x_{A 1}\right)}{L}\left(T_{A 2}-T_{A 1}\right) .
$$

On the condition of $T_{C}$ being known, the travel-time from $C$ to $P$ can be obtained 


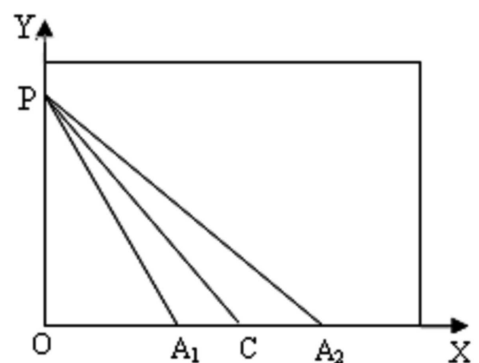

Fig. 1. Intersection schematic drawing of the boundary and rays.

$$
T_{P}=T_{C}+s \sqrt{\left(x_{P}-x_{C}\right)^{2}+\left(y_{P}-y_{C}\right)^{2}} .
$$

Substitute Eq. (1) into Eq. (2) as the following:

$$
\begin{gathered}
T_{P}=T_{A 1}+\frac{\left(x_{C}-x_{A 1}\right)}{L}\left(T_{A 2}-T_{A 1}\right) \\
+s \sqrt{\left(x_{P}-x_{C}\right)^{2}+\left(y_{P}-y_{C}\right)^{2}} .
\end{gathered}
$$

Supposing $r-x_{C}, \Delta T=T_{A 2}-T_{A 1}$, then

$$
\begin{aligned}
& T_{P}=T_{A 1}+\frac{\left(r-x_{A 1}\right)}{L}(\Delta T) \\
& \quad+s \sqrt{\left(x_{P}-x_{C}\right)^{2}+\left(y_{P}-y_{C}\right)^{2}} .
\end{aligned}
$$

The partial derivative of $T_{P}$ about $r$ is equal to zero. That is

$$
\frac{\partial T_{P}}{\partial r}=0 .
$$

We can archive

$$
r=x_{P}-\frac{\left(y_{P}-y_{C}\right) \Delta T}{\sqrt{L^{2} s^{2}-\Delta T^{2}}} .
$$

Substituting Eq. (6) into Eq. (4) further gives

$$
\begin{gathered}
T_{P}=T_{A 1}+\frac{\left(x_{P}-x_{A 1}\right)}{L} \Delta T \\
+\frac{\left(y_{P}-y_{C}\right)}{L} \sqrt{L^{2} s^{2}-\Delta T^{2}} .
\end{gathered}
$$

\section{Drawbacks of LTI algorithm}

Suppose the model $\mathrm{I}$ is a square which is divided into $3 \times 3$ grids. The middle grid is the low velocity area and the sonic velocity is $700 \mathrm{~m} / \mathrm{s}$. In other grids the sonic velocity is $3500 \mathrm{~m} / \mathrm{s}$. Model $\mathrm{I}$ is shown in Fig. 2 .

We only consider the condition that the launching point is 1 and the receiving points are 2,3 and 4 . The paths are obtained through LTI algorithm and shown in Fig. 3.

In Fig. 3 the real lines are paths of rays through LTI algorithm. The dashed line is the shortest path about travel-time from 1 to 3 point theoretically. That is to say paths solved by the LTI algorithm do not go round the low velocity area if the launching point, the receiving point and the low velocity area distribute on the same column. Otherwise, the other paths can go round the low velocity area.

Because the right grids of the column at which the launching point, the receiving point and the low velocity

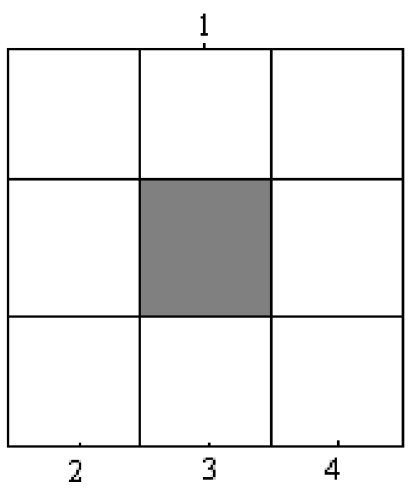

Fig. 2. Model I.

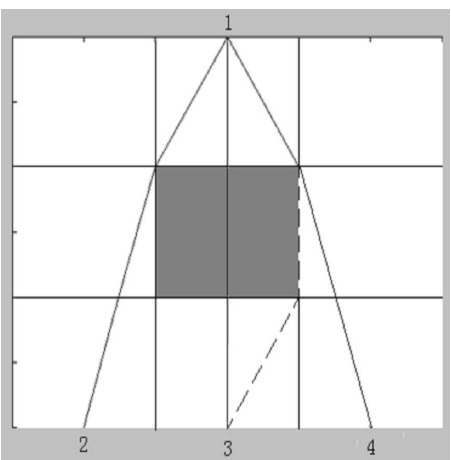

Fig. 3. Paths of model I.

area locate are scanned only toward right and the left grids are scanned only toward left, the path is always connected between the launching point and the receiving point. As a result, it is impossible to go round the low velocity area and the correct path about the minimum travel-time cannot be obtained.

The LTI algorithm has a drawback: rays cannot go round the low velocity area if the launching point, the receiving point and the low velocity area distribute on the same column (row). Aiming at this drawback, we have made improvement on LTI algorithm.

\section{Improvement of LTI algorithm}

The improved LTI algorithm is composed of two parts: forward processing and backward processing.

\subsection{Improvement of forward processing}

Equation (7) is the basic formula of forward processing. Using it, the minimum travel-time from the launching point to discrete points can be calculated when the distribution of discrete points in grids is known.

The steps of forward processing algorithm could be described as following.

(1) Suppose model II is divided into $3 \times 3$ grids as shown in Fig. 4. We calculate the minimum travel-time from the launching point $S$ to discrete points of the launching 


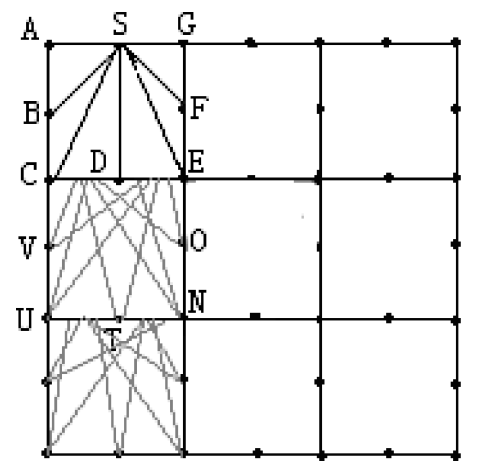

Fig. 4. Schematic diagram of forward processing steps (1) and (2).

point grid and regard these discrete points as the secondary launching sources.

If locations of $S, A, B, C, D, E, F$ and $G$ are known, the travel-time from $S$ to discrete points $A, B, C, D$, $E, F$ and $G$ can be deduced. According to Eq. (7), the minimum travel-time from $S$ to any point in the grid can be calculated.

(2) We calculate the minimum travel-time of all discrete points which are on boundaries of grids. These grids locate in the launching point grid's column. The corresponding discrete points are regarded as the secondary sources.

Firstly, travel-time of discrete points is calculated. The discrete points are on boundaries of the grid which is below the launching point grid. Here, the rays from the upper boundary $(C D E)$ are only considered.

For example, the minimum travel-time $t_{1}$ can be obtained from a point in $C D$ to $O$ point, the other minimum travel-time $t_{2}$ can be obtained from a point in $D E$ to $O$. So $t=\min \left(t_{1}, t_{2}\right)$ is regarded as the travel-time of $O$ point. The corresponding intersection point of boundary is recorded as the secondary source.

The process is repeating one by one. Finally travel-time of every discrete point is obtained. These discrete points are in boundaries of grids which are in the same column with the launching point grid.

(3) We calculate the minimum travel-time of all discrete points which are on boundaries of grids. These grids locate in the launching point grid's row. The method of calculation is the same as step (2). The corresponding discrete points are regarded as the secondary sources.

We start at the launching point grid's row. After obtaining travel-time of every discrete point which is on boundaries of the launching point grid, we carry out scanning from the right (left) one by one until the right (left) boundary of the model is finished. In the scanning process, the minimum travel-time and the secondary source should be amended if travel-time is smaller than the original travel-time. Otherwise the original value and the original secondary source should be retained. The process is shown as Fig. 5.

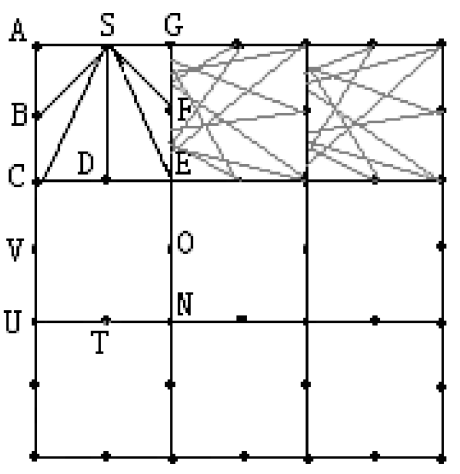

Fig. 5. Schematic diagram of forward processing step (3).

(4) The rays go toward only one direction. These rays are in grids which are in the same row or column with the launching point grid. In the other grids four directions are involved for the minimum travel-time.

The left boundary and upper boundary are taken for the grid which is in right bottom of the launching point or the secondary source. The left boundary and lower boundary are taken for the grid which is in right upper of the launching point or the secondary source. The right boundary and lower boundary are taken for the grid which is in left upper of the launching point or the secondary source. The right boundary and upper boundary are taken for the grid which is in left bottom of the launching point or the secondary source. Ten corresponding points are found separately through this method. The way of up and down about cross-scanning is applied to calculate travel-time.

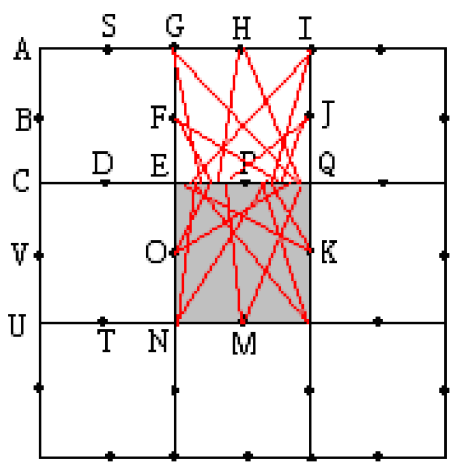

Fig. 6. Schematic diagram of cross-scanning about segment $E P Q$.

We assume that the right bottom grid of the launching point $S$ is at the intersection of No. 2 row and No. 2 column (shown as Fig. 6).

Ten points $(F, G, H, I, J, K, L, M, N$ and $O)$ around the upper boundary (segment $E P Q$ ) are applied to calculate travel-time (shown as Fig. 6).

Ten points $(D, C, V, U, T, M, L, K, Q$ and $P)$ around the left boundary (segment $E O N$ ) are applied 


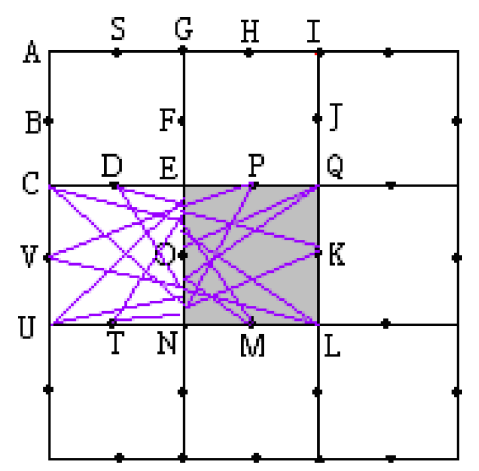

Fig. 7. Schematic diagram of cross-scanning about segment $E O N$.

to calculate travel-time by means of the method (shown as Fig. 7).

(5) On the basis of calculating travel-time of each grid, whole global travel-time which is from the launching points to the receiving points can be archived through adding up travel-time. The least global travel-time is the minimum travel-time.

\subsection{Simplified backward processing}

Equation (6) is the fundamental formula of ray tracing backward process. Its function is that the shortest path can be deduced when whole travel-time of boundaries in a grid is known.

The steps of backward processing algorithm could be described as following:

(1) The discrete point which is the minimum travel-time to the receiving point in the receiving point grid can be obtained through Eq. (6). The shortest path is the line of the discrete point and the receiving point.

Formula of travel-time $t_{i}$ is

$$
t_{i}=t^{\prime}+s \cdot d_{i} \text {. }
$$

$t^{\prime}$ is the travel-time which is obtained through forward process about a discrete point, $d_{i}$ is the distance from the discrete point to the receiving point, $s$ is the reciprocal of velocity. $s=1 / v . s$ is so-called slowness.

The minimum travel-time is $t=\min \left(t_{1}, t_{2}, \ldots, t_{i}, \ldots\right)$. We assume the discrete point $B^{\prime}$ exists in the receiving point grid. $t$ is the sum of travel-time from the launching point $S$ to $B^{\prime}$ and travel-time from point $B^{\prime}$ to the receiving point $R$ (shown as Fig. 8).

(2) According to the discrete point $B^{\prime}$ and the maximum gradient about the travel-time, we can find out the intersection point in neighboring segments.

We assume that the local minimum travel-time which is from a point in $A^{\prime} B^{\prime}$ to the receiving point $R$ is $t_{C 1}$. The point is $r_{1}$. The local minimum travel-time which is from a point in $B^{\prime} C^{\prime}$ to the receiving point $R$ is $t_{C 2}$. The point is $r_{2}$. If $t_{C 1}<t_{C 2}, r_{1}$ becomes the intersection point about the local minimum travel-time, otherwise $r_{2}$ becomes this intersection point. $r_{1}$ is the intersection point shown in Fig. 7.

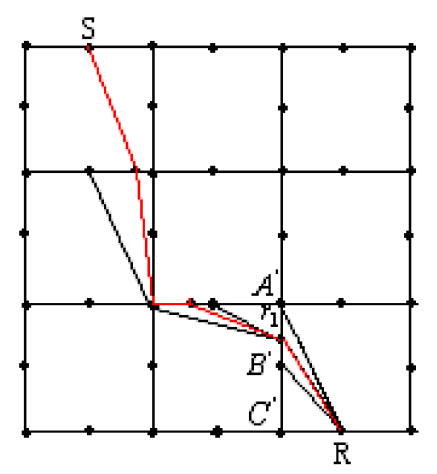

Fig. 8. Schematic diagram of backward processing.

(3) We regard the intersection point as a new receiving point and repeat steps (1), (2) until the launching point grid.

(4) We connect the launching point, all intersection points and the receiving point, the tracing path of rays is completed (shown as Fig. 7).

(5) Dealing steps (1)-(4) with all launching points and receiving points, all paths in materials can be traced.

The above is the realizable course of backward process.

Due to the introduction of Eq. (6), the calculation about paths is complex and line connecting is inconvenient in the course of backward processing. In order to solve the problem, we regard a discrete point directly as the secondary source to scan the other seven points in a grid. When the minimum travel-time is obtained, the corresponding discrete point is regarded as the next secondary source. The same method is used till the launching point.

In the course of realizing the algorithm, we will meet a case that four points are around a discrete point. There will be small change in the algorithm. All discrete points in neighboring grids are scanned. A discrete point of the minimum travel-time is regarded as the secondary source. Particularly there should be a course to judge stop otherwise the process will continue so as to fall into an endless loop.

The advantages of simplified backward processing are as follows: it simplifies the course of editing program, is benefit to line connecting. However, the simplified algorithm also brings loss of accuracy. Using the method of increasing in number of grids can avoid the drawback.

\section{Experimental results}

Suppose the model I is a square which is divided into $3 \times 3$ grids (shown as Fig. 2). It is 3 meters in length and 3 meters in width. The middle grid is the low velocity area and the sonic velocity is $700 \mathrm{~m} / \mathrm{s}$. In other grids the sonic velocity is $3500 \mathrm{~m} / \mathrm{s}$. The tracing paths of model I which is obtained through the improved LTI algorithm is shown in Fig. 9.

From Fig. 9 we can see that the tracing path about the minimum travel-time from point 1 to point 3 is composed 


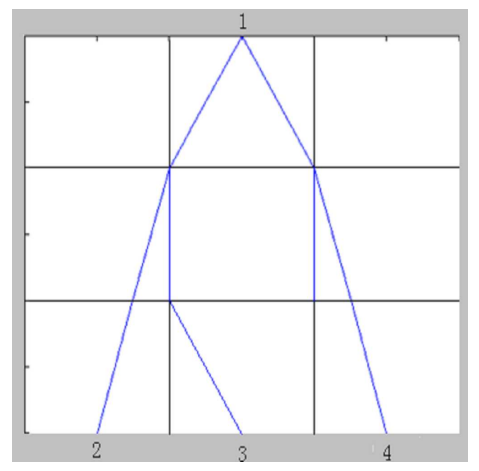

Fig. 9. Linear interpolation ray tracing paths of model I.

of the oblique line in the launching point unit, the left boundary of the low velocity area, and the oblique line in the receiving point unit.

TABLE

Comparison of the minimum travel-time.

\begin{tabular}{c|c|c|c}
\hline \hline & $\begin{array}{c}\text { The minimum } \\
\text { travel-time } \\
\text { from point 1 } \\
\text { to point 2 }\end{array}$ & $\begin{array}{c}\text { The minimum } \\
\text { travel-time } \\
\text { from point 1 } \\
\text { to point 3 }\end{array}$ & $\begin{array}{c}\text { The minimum } \\
\text { travel-time } \\
\text { from point 1 } \\
\text { to point 4 }\end{array}$ \\
\hline $\begin{array}{c}\text { LTI algorithm } \\
\text { The improved }\end{array}$ & $9.1652 \times 10^{-4}$ & 0.0031 & $9.1652 \times 10^{-4}$ \\
$\begin{array}{c}\text { LTI algorithm } \\
9.1652 \times 10^{-4}\end{array}$ & $9.2459 \times 10^{-4}$ & $9.1652 \times 10^{-4}$
\end{tabular}

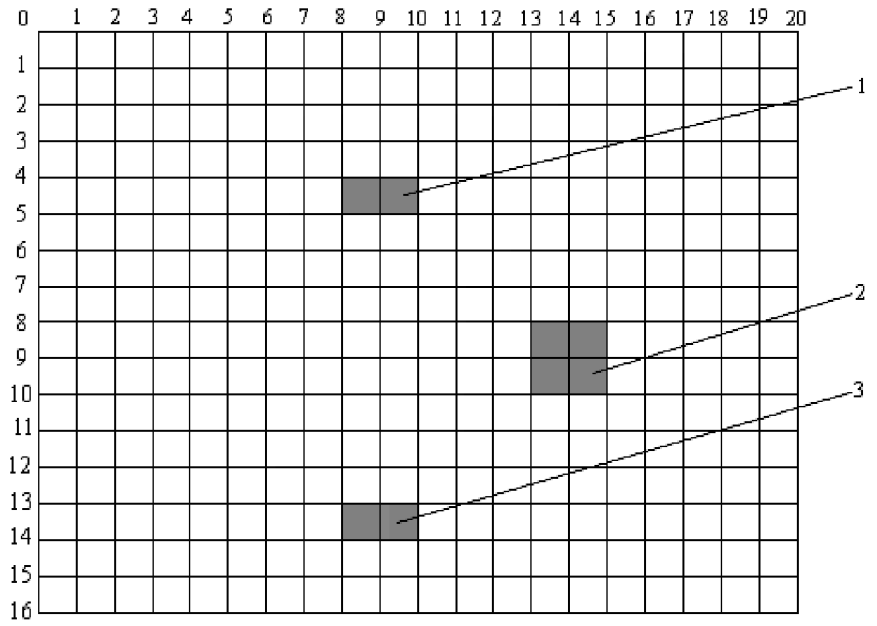

Fig. 10. Model III: 1 - the first low velocity area, 2 the second low velocity area, 3 - the third low velocity area.

The minimum travel-time from the launching point to the receiving points through the LTI algorithm and the improved LTI algorithm is showed in Table. The minimum travel-time from the launching point 1 to the receiving point 3 though the improved LTI algorithm is far

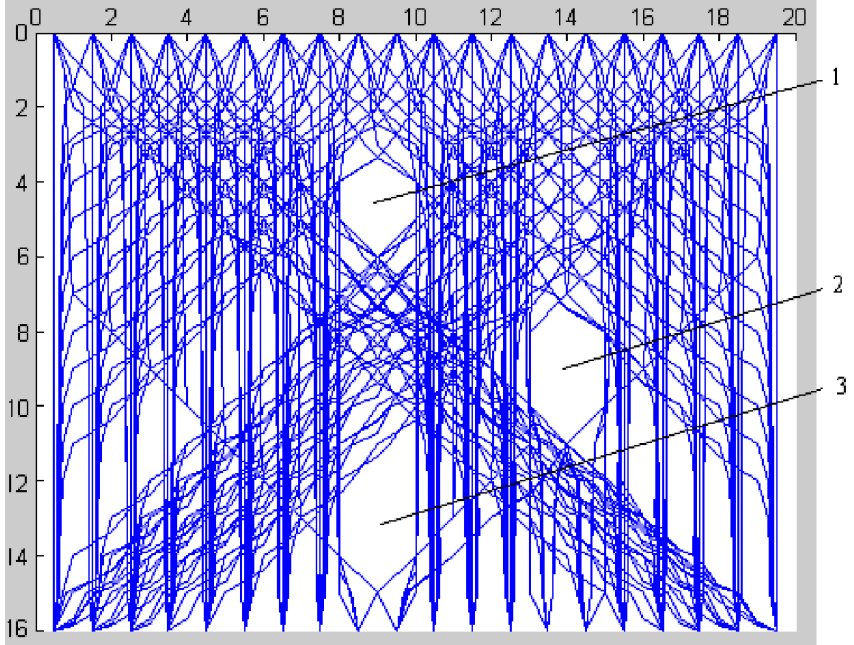

Fig. 11. Paths of model III: 1 - the first low velocity area, 2 - the second low velocity area, 3 - the third low velocity area.

smaller than that of the LTI algorithm. The minimum travel-time of the improved LTI algorithm approaches the theoretical value.

Model III is shown in Fig. 10. Its entire region is divided into $20 \times 16$ grids. In this model the black region stands for low velocity areas, the sonic velocity is $800 \mathrm{~m} / \mathrm{s}$. The sonic velocity of other region is $3200 \mathrm{~m} / \mathrm{s}$. Ultrasonic is set from the first row and received by the last row.

The paths of adopting the improvement on ray tracing algorithm have been shown in Fig. 11.

In Fig. 11 we can see when launching points, receiving points and low velocity areas are not in the same columns, the paths which are obtained through the improvement of LTI algorithm can go round three low velocity areas. When launching points, receiving points and low velocity areas are in the same columns, paths can also go round low velocity areas.

\section{Conclusion}

The improved linear interpolation ray tracing algorithm adopts a cross-scanning strategy to carry out forward processing and uses discrete points as the secondary sources to simplify backward processing. The rays can go round low velocity areas located in the same column (row) of launching points and receiving points. The experiment shows that the improvement of LTI algorithm is correct and effective.

\section{Acknowledgments}

Supported by the National Natural Science Foundation of China under grant No. 60372073. 


\section{References}

[1] G. Bernasconi, G. Drufuca, Geophysics 66, 277 (2001).

[2] H.J.A. Van Avendonk, A.J. Harding, J.A. Orcutt, W.S. Holbrook, Geophysics 66, 648 (2001).

[3] T. Watanabe, K. Sassa, Int. J. Rock Mech. Min. Sci. Geomech. Abstr. 36, 467 (1996).

[4] F. Haslinger, E. Kissling, Phys. Earth Planet. Interiors 123, 103 (2001).

[5] M. Krause, F. Mielentz, B. Milman, W. Muller, V. Schmitz, H. Wiggenhauser, NDTEE International 34, 403 (2001).

[6] E. Cardarelli, A. Cerreto, Geophysical Prospecting 50, 55 (2002).
[7] Z. Yun-shu, G. Han-ming, S. Xue-min, Chin. J. Eng. Geophys. 2, 167 (2005).

[8] D. Cores, G.M. Fung, R.J. Michelena, J. Appl. Geophys. 45, 273 (2000).

[9] F. Lingvall, Ultrasonics 42, 961 (2004).

[10] H. Gemmeke, N.V. Ruiter, Nucl. Instrum. Methods Phys. Res. A 580, 1057 (2007).

[11] E. Asakawa, T. Kawanaka, Geophysical Prospect 41.1, 99 (1993).

[12] J.E. Vidale, Geophysics 55, 521 (1990). 